Research Paper

\title{
Systemic Deficiency of GHR in Pigs leads to Hepatic Steatosis via Negative Regulation of AHR Signaling
}

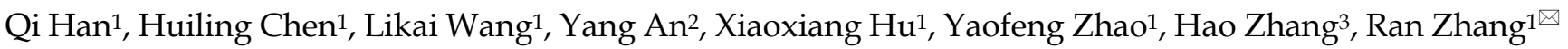 \\ 1. State Key Laboratory of Agrobiotechnology, College of Biological Sciences, China Agricultural University, Beijing, 100193, China. \\ 2. MD Department of Plastic Surgery, Peking University Third Hospital, Beijing, 100191, China. \\ 3. National Engineering Laboratory for Animal Breeding, China Agricultural University, Beijing, 100193, China.
}

$\triangle$ Corresponding author: Ran Zhang, State Key Laboratory of Agrobiotechnology, College of Biological Sciences, China Agricultural University, Beijing, 100193, China. Telephone: 86-10-62731327; E-mail: zhangran0628@cau.edu.cn.

(C) The author(s). This is an open access article distributed under the terms of the Creative Commons Attribution License (https://creativecommons.org/licenses/by/4.0/). See http://ivyspring.com/terms for full terms and conditions.

Received: 2021.07.13; Accepted: 2021.09.23; Published: 2021.10.03

\begin{abstract}
Laron syndrome (LS) is an autosomal recessive genetic disease mainly caused by mutations in the human growth hormone receptor (GHR) gene. Previous studies have focused on Ghr mutant mice, but compared with LS patients, Ghr knockout (KO) mice exhibit differential lipid metabolism. To elucidate the relationship between GHR mutation and lipid metabolism, the role of GHR in lipid metabolism was examined in GHR KO pigs and hepatocytes transfected with siGHR. We observed high levels of free fatty acids and hepatic steatosis in GHR KO pigs, which recapitulates the abnormal lipid metabolism in LS patients. RNAseq analysis revealed that genes related to the fatty acid oxidation pathway were significantly altered in GHR KO pigs. AHR, a transcription factor related to lipid metabolism, was significantly downregulated in GHR KO pigs and siGHR-treated human hepatocytes. We found that AHR directly regulated fatty acid oxidation by directly binding to the promoters of ACOX 1 and CPTIA and activating their expression. These data indicate that loss of GHR disturbs the ERK-AHR-ACOX1/CPTIA pathway and consequently leads to hepatic steatosis. Our results established AHR as a modulator of hepatic steatosis, thereby providing a therapeutic target for lipid metabolism disorder.
\end{abstract}

Key words: Laron syndrome; GHR; hepatic steatosis; AHR

\section{Introduction}

Laron syndrome (LS), also known as growth hormone insensitivity syndrome, is an autosomal recessive genetic disease associated with severe postpartum growth retardation [1]. Single gene deletion of GHR or a lack of the molecule downstream of the receptor in the GH signalling pathway are the causes of LS. Among these causes, GHR mutation is the most common genetic aberration $[2,3]$. GH binds to dimeric GHR to induce its conformational rotation, which leads to the activation of different signalling pathways, including the JAK2-STATs [4], MAPK [5] and PI3K-AKT [6] pathways, to promote the production of IGF1 in liver tissues and mediate growth promotion and metabolic regulation processes $[7,8]$. This phenomenon predisposes individuals with LS to manifestations of hepatic steatosis [9]. However, the mechanisms linking LS and hepatic steatosis remain poorly understood. Thus, it is crucial to improve our understanding of the pathophysiology of LS and its comorbidities.

The pathogenesis of LS induced by GHR mutation is currently explored through systematic or conditional knockout $(\mathrm{KO})$ of $\mathrm{Ghr}$ in various tissues and organs of mice [10-15]. However, the effect of GHR mutation on lipid metabolism differs between mice and humans. LS patients with increased serum levels of free fatty acids (FFAs) often develop fatty liver; however, mice carrying a global Ghr KO show normal serum levels of FFAs and do not develop hepatic steatosis. Therefore, this mouse model cannot fully simulate human LS and cannot be used to draw conclusions about the lipid metabolism in human LS [16]. Minipigs, mammals with an adult weight of approximately 30 kilograms are small in size and easy to handle. Moreover, lipid metabolism in minipigs is more similar to that in humans, and fat digestion and 
absorption in minipigs are similar to those in humans $[17,18]$. Additionally, changes in blood lipids in minipigs fed a high-fat diet are consistent with those observed in obese humans [19]. Therefore, minipigs are more suitable than mice as for modelling human lipid metabolism-related diseases [20,21].

Here, we established a GHR KO pig model [22] to investigate the cause of abnormal lipid metabolism. Initially, we found that deletion of GHR in pigs resulted in an imbalance in glucose and lipid metabolism, culminating in marked hepatic steatosis. Transcriptome analysis showed significant changes in signals related to lipid metabolism. Subsequently, we used siGHR-transfected human hepatocytes to confirm that GHR deletion affected affect expression of the key fatty acid oxidation enzymes ACOX1 and CPT1A by reducing the expression of AHR, which triggered abnormal fatty acid degradation and led to fat deposition. Our findings revealed the important role of liver GHR in lipid metabolism.

\section{Materials and methods}

\section{Animals}

GHR KO pigs on the China Experimental Mini Pigs background were created using the zinc finger nuclease (ZFN) system. Pig genotyping was performed by PCR with the primers $5^{\prime}$-AAGCG GTGTCTATGTGCTGATTCTC- $3^{\prime}$ and $5^{\prime}$-TCAGTGG CTAGAGTATATGATGTTG- $3^{\prime}$; the WT and targeted alleles produced PCR bands of $530 \mathrm{bp}$ and $534 \mathrm{bp}$, respectively. The mRNA level of the GHR gene was confirmed by Q-PCR, and the protein level of GHR was evaluated by Western blotting and immunohistochemistry with an anti-GHR antibody (Bioss, China).

\section{Cell culture}

HepG2 and L02 cells were maintained in our laboratory. These cells were maintained in Dulbecco's modified Eagle's medium (DMEM, Gibco, Grand Island, NY, USA) supplemented with $10 \%$ heatinactivated foetal bovine serum (FBS) at $37{ }^{\circ} \mathrm{C}$ in a humidified atmosphere of $5 \% \mathrm{CO}_{2}$.

Hepa1-6 cells were purchased from Procell and were maintained in DMEM supplemented with $10 \%$ heat-inactivated FBS, $1 \mathrm{mM}$ sodium pyruvate and 1\% penicillin/streptomycin $(\mathrm{P} / \mathrm{S})$ at $37^{\circ} \mathrm{C}$ in a humidified atmosphere of $5 \% \mathrm{CO}_{2}$.

\section{Plasmid construction}

The promoter sequence of ACOX1 was cloned into the pGL3-Basic vector to construct the pGL3ACOX1 plasmid. The pGL3-CPT1A was constructed similarly. The coding sequence (CDS) of $A H R$ was cloned into the pCMV-Myc vector to construct
pCMV-Myc-AHR.

\section{GHR siRNA treatment in hepatocytes}

GHR knockdown cells were established by using an siRNA directed against GHR. HepG2, L02 and Hepa1-6 cells were transfected with siGHR using Lipofectamine 2000 transfection reagent (Life Technologies, Grand Island, NY) in accordance with the manufacturer's instructions. First, cells were cultured in 12-well plates overnight. Then, $40 \mathrm{pmol}$ negative control (NC) siRNA or siGHR mixed with Lipofectamine was added to each well. The cells were collected after 24 hours for follow-up experiments.

\section{AHR siRNA treatment in hepatocytes}

AHR knockdown cells were established by using an siRNA directed against AHR. HepG2 were transfected with siAHR using Lipofectamine 2000 transfection reagent (Life Technologies, Grand Island, NY) in accordance with the manufacturer's instructions. First, cells were cultured in 12-well plates overnight. Then, 40 pmol negative control (NC) siRNA or siAHR mixed with Lipofectamine was added to each well. The cells were collected after 24 hours for follow-up experiments.

\section{Analysis of plasma metabolites}

Plasma glucose, insulin, TGs, TC, HDL, LDL, FFAs, ALT and AST were measured by Beijing Zhongtong Lanbo Clinical Laboratory.

\section{Intravenous glucose tolerance test (IGTT)}

After fasting blood glucose levels were measured, each pig was injected intravenously with $1.2 \mathrm{ml}$ of a $50 \%$ glucose solution per kilogram body weight $\left(\mathrm{ml} \mathrm{kg}^{-1}\right)$. Blood glucose levels were then measured at $0,2,5,10,20,30,60$ and 120 minutes post injection using a glucometer.

\section{Measurement of the TG content in liver tissue and cells}

Livers collected from the pigs were stored at -80 ${ }^{\circ} \mathrm{C}$ until use. The TG content in the liver and hepatocytes was determined using a Triglyceride Assay Kit according to the manufacturer's protocol (Nanjing Jiancheng Bioengineering Institute, China). The protein concentration was determined with a BCA Protein Assay Kit (Beyotime Biotechnology, China).

\section{Q-PCR}

Total RNA was extracted with TRIzol (Invitrogen, USA), and reverse transcription was performed with $1 \mu \mathrm{g}$ of total RNA using PrimeScript ${ }^{\mathrm{TM}}$ RT Master Mix (TaKaRa, Japan). SYBR Green PCR Master Mix (Roche, Switzerland) was 
used for PCR amplification. All reactions were performed in triplicate. The mRNA expression levels of the target genes were normalized to that of GAPDH. The primers used are listed in Supplementary Table S1.

\section{Western blot analysis}

Total cell lysates were prepared from either liver tissue or cultured cells by lysis using RIPA Lysis Buffer (Beyotime, China) containing 1\% PMSF and homogenization using a vortex oscillator (Roche, USA). Protein concentrations were determined using an Enhanced BCA Protein Assay Kit (Beyotime, China). An equal volume of $5 X$ loading buffer was mixed with the samples, which were boiled for 10 minutes. After separation by 10\% SDS-PAGE, the proteins were transferred to a $0.45 \mathrm{~mm}$ PVDF membrane and blocked for 2 hours with $5 \%$ non-fat dry milk at room temperature. Then, the membrane was incubated overnight at $4{ }^{\circ} \mathrm{C}$ with the appropriate primary antibody. After incubation with the secondary antibody for another hour, the membranes were developed with SuperSignal ${ }^{\mathrm{TM}}$ West Pico PLUS Chemiluminescent Substrate (Thermo, USA). The following primary antibodies were used. Antibodies against ACOX1 (A8091) and CPT1A (A5307) were purchased from ABclonal, and an antibody against AHR (\#4685) was purchased from Cell Signaling Technology (Danvers, MA, USA). The antibody against GAPDH (AG019) was purchased from Beyotime Biotechnology.

\section{Histopathological, IHC and immunofluorescence (IF) staining}

Tissues were fixed with $4 \%$ paraformaldehyde and then sequentially dehydrated in increasing concentrations of ethanol ranging from 75 to $100 \%$. Dehydrated specimens were subsequently cleared with xylene, embedded in paraffin, processed for paraffin sectioning (5-mm sections), and subjected to H\&E, IHC or IF staining. For IHC staining, sections were incubated overnight with primary antibodies and then with HRP-conjugated secondary antibodies. For IF staining, the sections were incubated overnight with primary antibodies and then with Alexa Fluor ${ }^{\circledR}$ 594/488-conjugated secondary antibodies and DAPI (Sigma).

\section{Oil red $O$ staining}

Samples of liver were embedded in O.C.T. compound (Solarbio China). Fresh frozen specimens were cryosectioned at a thickness of $6 \mu \mathrm{m}$ and air dried. The sections were then fixed in PBS for 5 minutes and, after soaking for 5 minutes in $60 \%$ isopropanol, stained with a $3: 2$ mixture of $0.5 \%$ Oil red $\mathrm{O}$ solution in isopropanol and distilled water for
15 minutes in the dark. The slides were washed with $60 \%$ isopropanol for $10 \mathrm{~s}$ and then washed with distilled water. Nuclei were then stained with haematoxylin for 5 minutes, bluing was performed with tap water, and the sections were sealed with glycerol-gelatine and observed under a microscope.

\section{Nile red staining}

Nile red staining was used for cellular lipid droplet staining. The cells to be tested were washed twice with PBS for 3 minutes each, fixed with $4 \%$ paraformaldehyde for 20 minutes, washed twice with PBS for 3 minutes each, stained with Nile red at $37^{\circ} \mathrm{C}$ for 20 minutes, and then washed with PBS 2 times for 3 minutes each. DAPI was used to stain the nuclei for 20 minutes, and the cells were finally washed twice with PBS and photographed under a microscope.

\section{Luciferase reporter assay}

L02 cells were plated in a 24 -well culture plate and transfected with a reporter vector (500 ng) together with each indicated expression plasmid using Lipofectamine 2000 (Invitrogen) according to the manufacturer's instructions. Luciferase activities were measured using a Dual Luciferase Reporter Assay System (Promega, Madison, WI, USA) according to the manufacturer's instructions. Firefly luciferase activity was normalized to Renilla luciferase activity.

\section{Chip assay}

L02 cells were plated in a $10 \mathrm{~cm}^{2}$ culture plate. After incubation with the AHR agonist Tapinarof (Topscience, T4644), for $12 \mathrm{~h}$, the cells were collected, and a ChIP assay was performed using a SimpleChIP Enzymatic Chromatin Immunoprecipitation Kit (Cell Signaling Technology, \#9003) according to the manufacturer's protocol. The primers used for realtime PCR analysis of the DNA fragments of interest among the immunoprecipitated DNA fragments are listed in Supporting Table S.

\section{CO-IP}

Pig livers and human hepatocytes were used to study whether GHR and AHR have a related effect. To the human hepatocytes was added AHR ligand Tapinarof. The cells were harvested after 24 hours, and the IP experiment was conducted according to Beyotime's kit. The final product was verified by Western blot analysis.

\section{Statistical analysis}

Data are expressed as the means \pm SDs and were analysed using GraphPad Prism Software 7.00. For comparisons between two groups of data that showed normal distribution and homogeneity of variance, the 
two-tailed Student's t-test was performed. P-values $<0.05$ were considered statistically significant.

\section{Results}

\section{Knockout of GHR in pig results in a severe dwarf phenotype}

To determine the physiological roles of GHR in vivo, we generated GHR KO pigs (Figure 1A). The
mRNA expression of GHR was markedly reduced in GHR KO pigs compared with wild-type (WT) pigs (Figure 1D), and the protein expression of GHR was downregulated in liver samples from GHR KO pigs (Figure 1E-F). In addition, immunohistochemical (IHC) staining of liver sections further indicated that the expression of GHR was decreased in the livers of GHR KO pigs (Figure 1G-H). We measured the growth rate of GHR $\mathrm{KO}$ pigs and observed that the
A

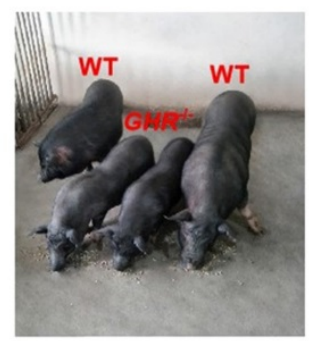

D

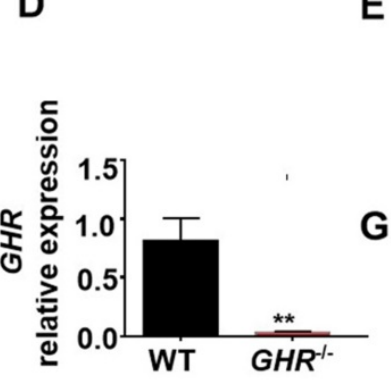

E

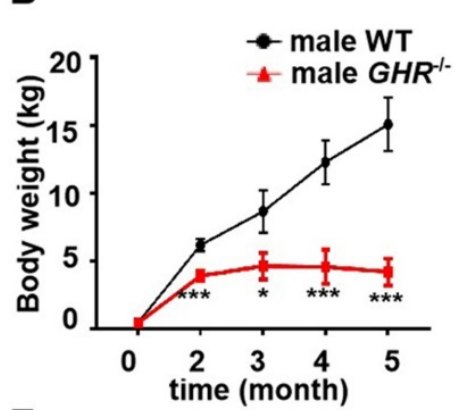

WT GHR $^{\text {-. }}$
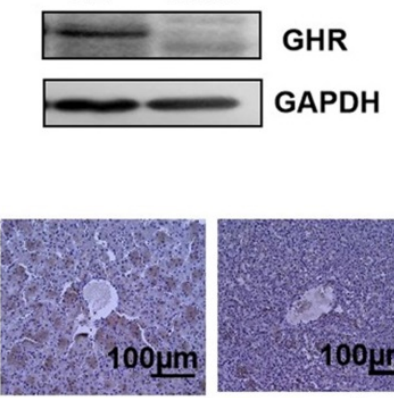

WT

I
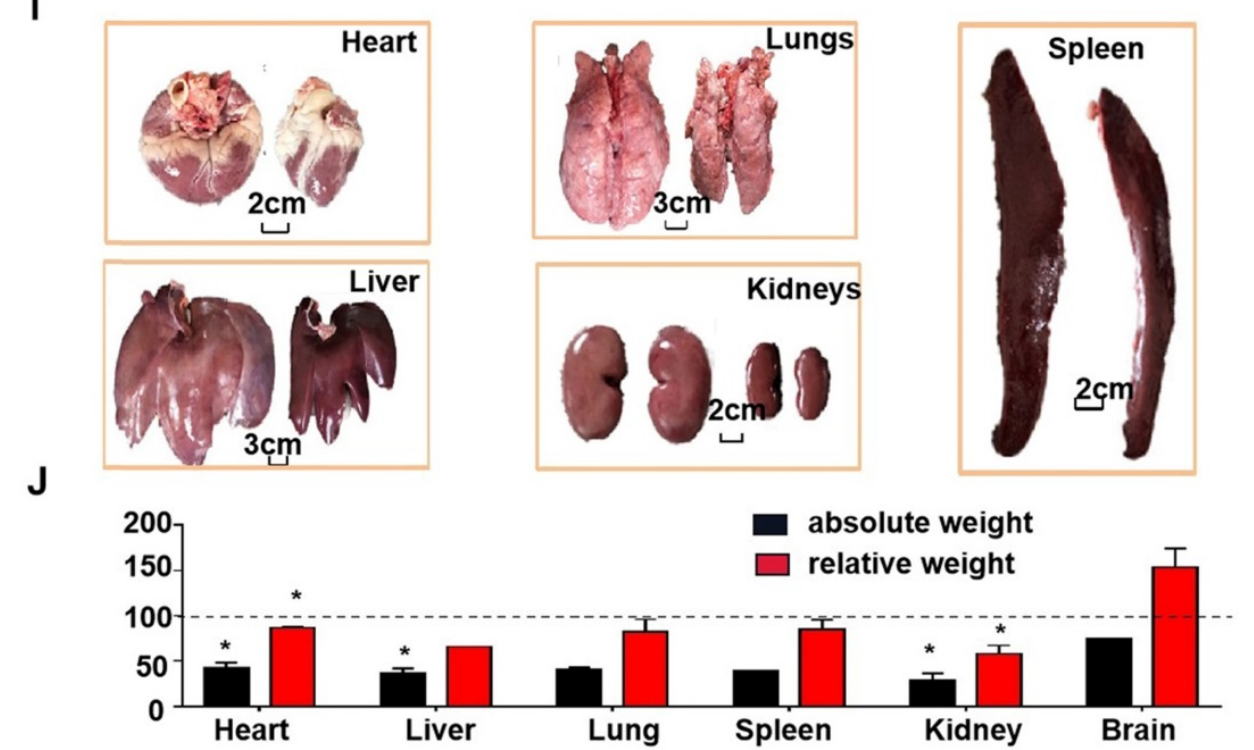

$\mathbf{J}$

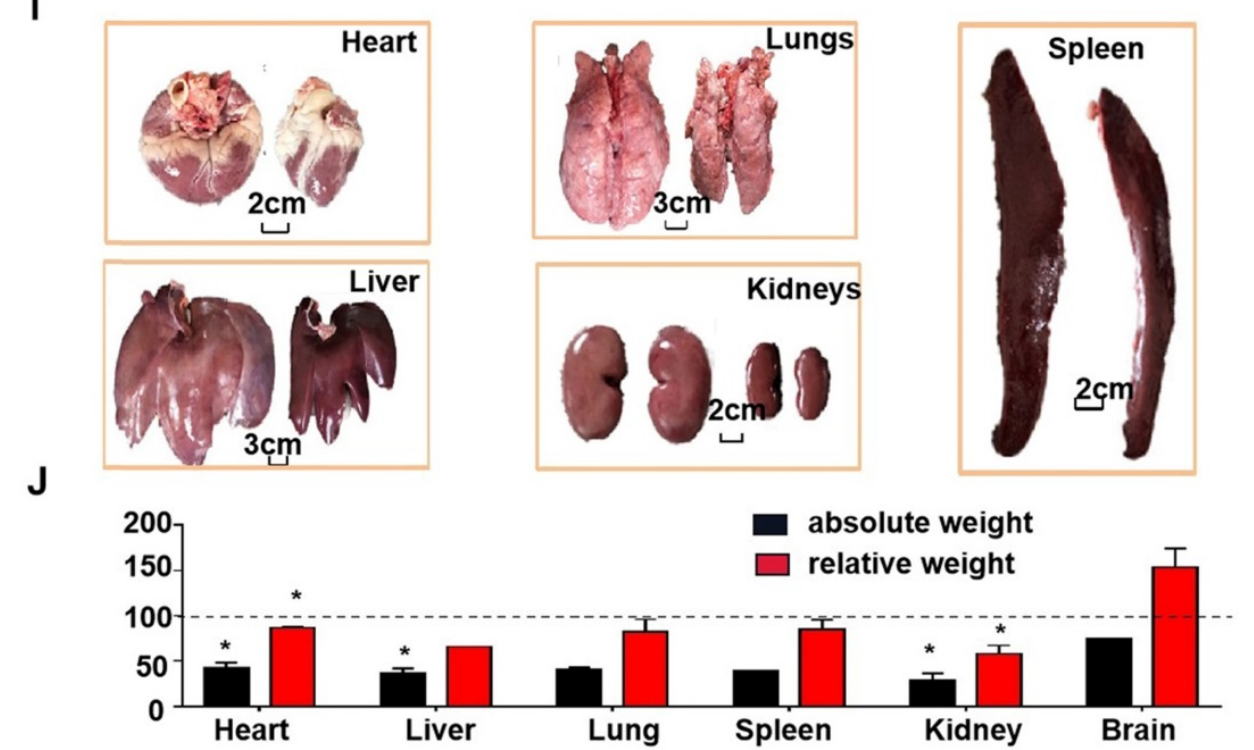

C

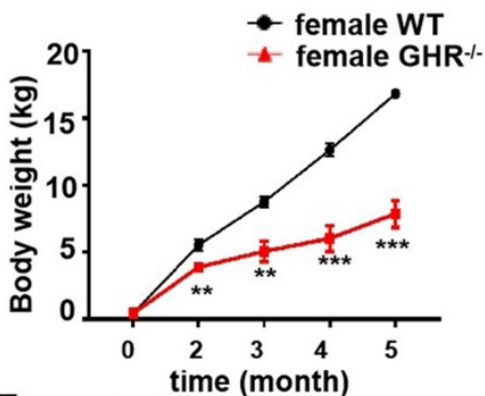

$\mathbf{F}$

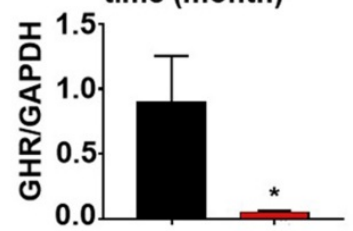

H

WT $G H R^{-1-}$

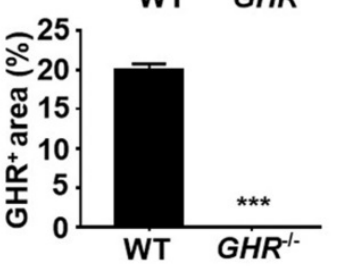

Figure 1. Phenotype of GHR KO pigs. (A) Representative photographs of pigs of the two genotypes. (B-C) Body weight of male and female GHR KO pigs and their WT littermates. (D-F) Hepatic GHR mRNA and protein levels in GHR KO pigs. (D) Hepatic GHR mRNA levels in GHR KO pigs. (E-F) Hepatic GHR protein levels in GHR KO pigs by Western blotting, and quantification of the content by Image). (G-H) Hepatic GHR protein levels in GHR KO pigs by IHC and quantification of the content by Image. (I) Representative photographs of organs from GHR KO pigs and their WT littermates. (J) Absolute weight and relative weight of the organs. Scale bar: $100 \mu \mathrm{m} . \mathrm{n}=3$ pigs per group. The data are presented as the mean \pm SD values. $*, P<0.05 ; * *, P<0.01 ; * * *, P<0.001$. Annotation: relative weight, the ratio of organ weight to body weight. 
weights of both male and female GHR KO pigs were approximately half those of WT pigs (Figure 1B-C), and the lengths were also significantly reduced (Supplementary Figure 1A-B). However, heterozygous GHR mutant pigs did not show any significant difference in GHR protein level, body weightor body length (Supplementary Figure 1C-F). Consistent with their smaller size, GHR KO pigs had a lower absolute weight of all organs than WT pigs. After normalization to the whole body weight, the heart, liver, spleen, lung, and kidney also showed significantly reduced relative weights, but the relative brain weights were increased in GHR KO pigs (Figure 1G-H).

\section{GHR deficiency disrupts glucose/lipid homeostasis in pigs}

GH can increase hepatic glucose production [23], and GH-GHR signaling plays a major role in regulating glucose metabolism [24]. Thus, we investigated the effect of GHR deficiency on glucose metabolism and observed that fasting blood glucose was significantly decreased in GHR $\mathrm{KO}$ pigs (Supplementary Figure 2A). In addition, the serum insulin level and homeostatic model assessment of insulin resistance (HOMA-IR) index were significantly lower in GHR KO pigs (Supplementary Figure 2B-C). The homeostatic model assessment of insulin sensitivity (HOMA-IS) index was significantly higher in GHR KO pigs (Supplementary Figure 2D). When glucose was administered by i.p. injection, GHR
KO pigs exhibited glucose intolerance (Supplementary Figure 2E-F). PI3K-AKT is the main downstream signaling pathway of insulin. Activated AKT increases glycogen synthesis and inhibits gluconeogenesis, thereby reducing blood sugar and increasing insulin sensitivity [25]. Therefore, Western blotting was used to detect AKT molecules. The results showed that the phosphorylation of AKT was significantly increased in GHR KO pigs (Supplementary Figure 2G-H), which could explain the insulin sensitivity symptoms of GHR KO pigs. Similarly, we used GHR KO pigs to confirm the function of GHR in lipid metabolism. Biochemical analysis showed large decreases in triglycerides (TGs), total cholesterol (TC), high-density lipoprotein (HDL) and low-density lipoprotein (LDL) in GHR KO pigs (Figure 2A-F). Notably, the serum FFA level in GHR KO pigs was significantly increased, consistent with the results in LS patients (Figure 2G). In summary, these findings indicate that disruption of GHR has a major effect on glucose and lipid homeostasis and that the resulting phenotype is more similar to that observed in human patients.

\section{GHR deficiency induces hepatic steatosis in pigs}

Owing to the critical role of GHR in lipid metabolism, we focused mainly on whether GHR deficiency affects hepatic steatosis in pigs. Biochemical analysis confirmed a significant increase in TGs in the livers of GHR KO pigs (Figure 3B),
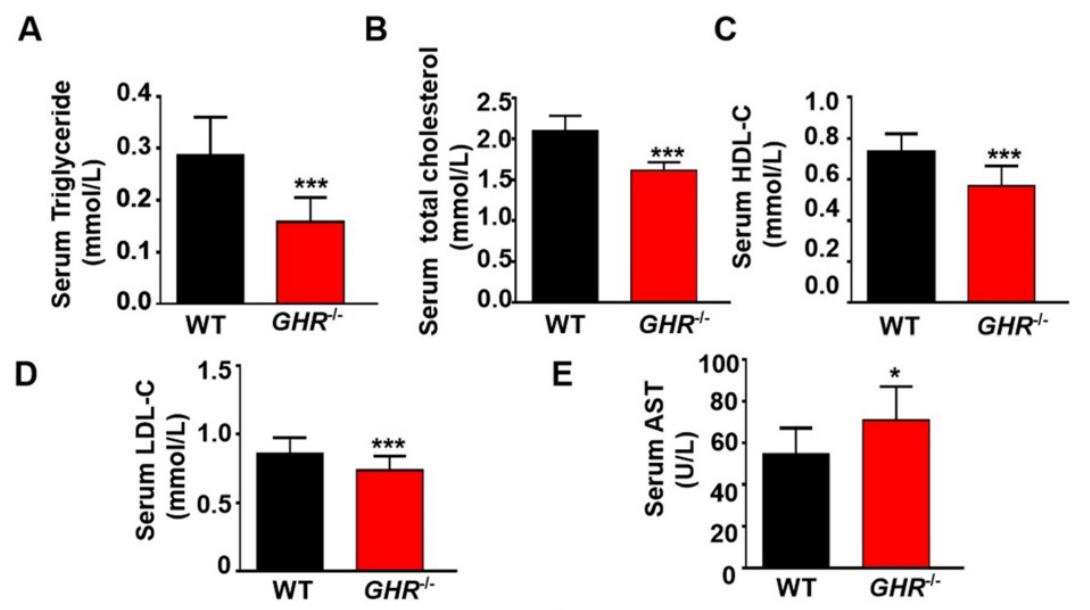

E

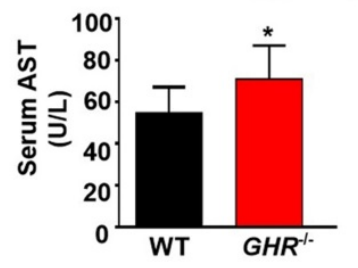

$\mathbf{F}$

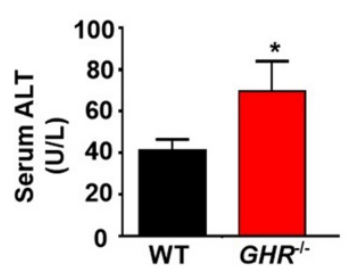

G

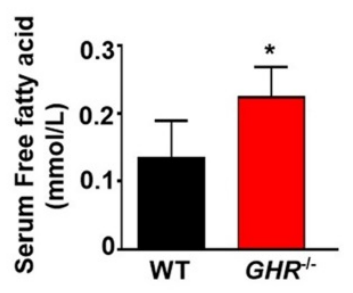

Figure 2. GHR deficiency disrupted lipid homeostasis in pigs. (A-E) Serum TG, TC, HDL, LDL and FFA levels in WT and GHR KO pigs. (F-G) Liver function markers, including ALT and AST, in WT and GHR KO pigs. $\mathrm{n}=3$ pigs per group. The data are presented as the mean \pm SD values. *, $\mathrm{P}<0.05 ; * *, \mathrm{P}<0.01 ; * * *, \mathrm{P}<0.001$. 
accompanied by increases in alanine aminotransferase (ALT) and aspartate aminotransferase (AST) suggestive of liver damage (Figure 2E-F). Accordingly, histological analyses of livers based on haematoxylin and eosin (H\&E) staining revealed increased numbers and sizes of intracellular vacuoles (an indication of increased fat deposition) in GHR KO pigs (Figure 3A). Oil red O (ORO) staining of liver sections verified the deposition of increasing quantities of lipids in GHR $\mathrm{KO}$ pigs (Figure 3C-D). The above results suggest that GHR deficiency leads to hepatic steatosis in pigs.

\section{GHR deficiency reduces fatty acid oxidation in pigs}

A series of studies was conducted to identify the source of hepatic steatosis in GHR KO pigs. The mRNA expression of genes responsible for fatty acid oxidation was significantly downregulated in GHR
KO pigs (Figure 3E). In particular, ACOX1 and CPT1A are the two rate-limiting enzymes of fatty acid oxidation [26]. The IHC results showed that the protein levels of ACOX1 and CPT1A were lower in GHR KO pigs (Figure 3F-G). Moreover, MTTP and APOB, two important indicators of VLDL secretion, were also significantly downregulated in GHRKO pigs. (Figure 3E), although the mRNA levels of fatty acid uptake-, synthesis- and inflammation- related genes were not significantly different (Supplementary Figure 3A). Taken together, these lines of evidence suggest that GHR deletion results in hepatic steatosis. Notably, GHR KO led not only to lipid accumulation in the pig liver but also to significant accumulation of lipids in the kidney, skeletal muscle and pancreas. Additionally, the size of adipocytes in GHR KO pigs increased significantly (Supplementary Figure 4).
A
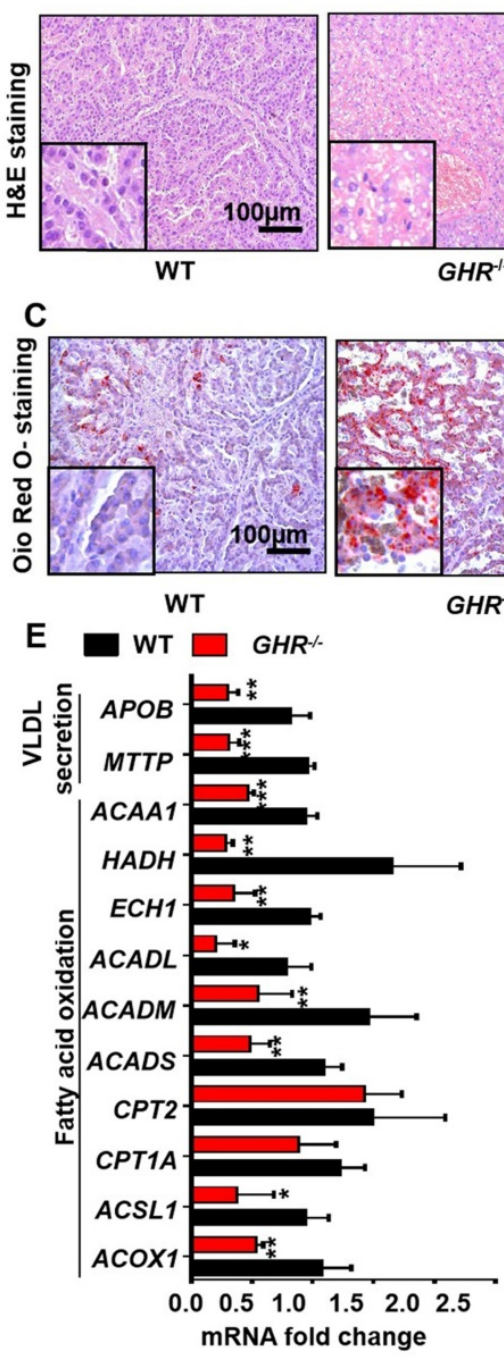

B

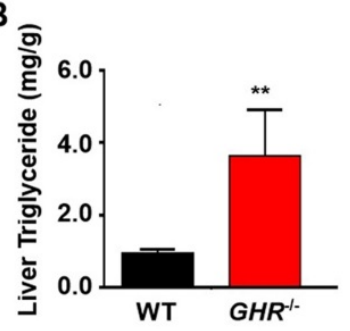

D

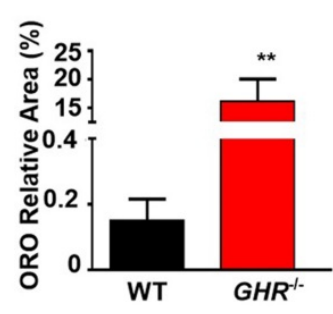

$F$
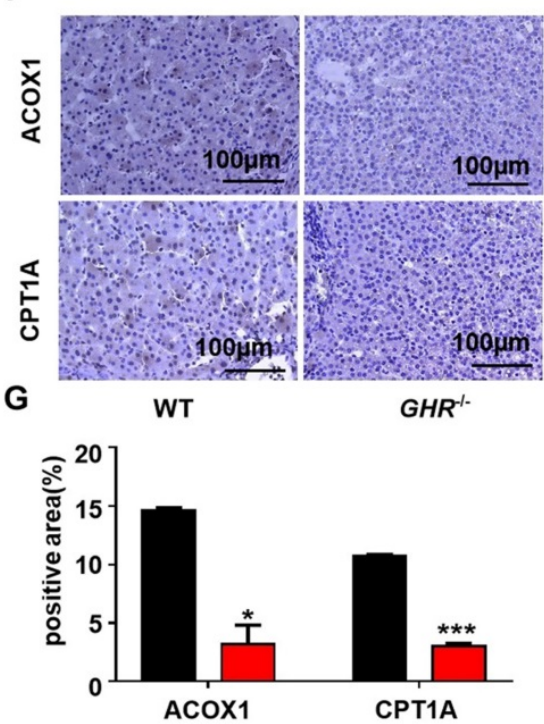

Figure 3. GHR KO pigs developed hepatic steatosis. (A) Representative image of H\&E staining. (B) Hepatic TG levels in WT and GHR KO pigs. (C-D) Representative image of Oil red $O$ staining and relative Oil red O-stained area. (E) mRNA levels of key genes in fatty acid oxidation and VLDL secretion. (F-G) Protein levels of key genes in fatty acid oxidation and quantification of the content by ImageJ. Scale bar: $100 \mu \mathrm{m} . \mathrm{n}=3$ pigs per group. The data are presented as the mean \pm SD values. $*, P<0.05 ; * *, P<0.01$; $* * *, \mathrm{P}<0.001$. 
A

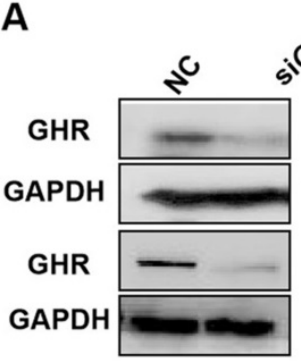

D
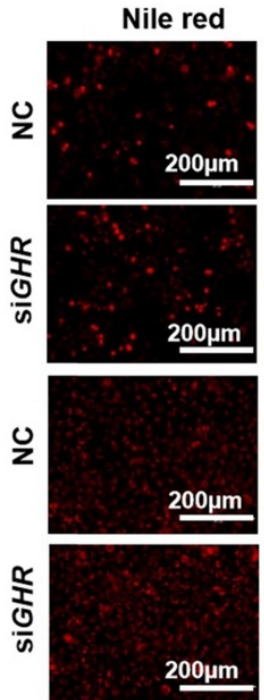

B

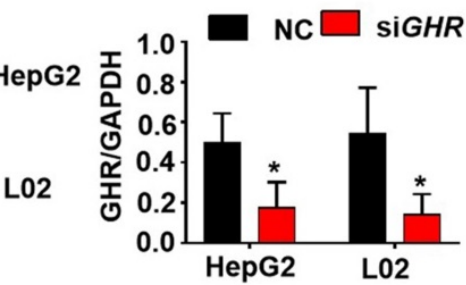

C

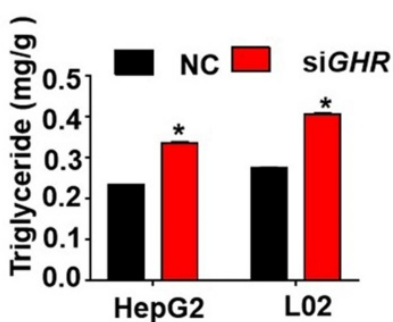

E

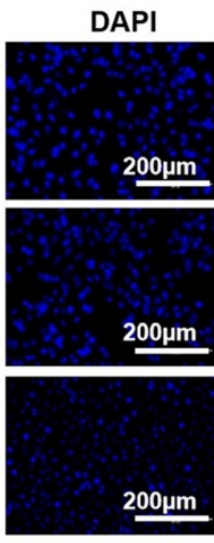

Merge
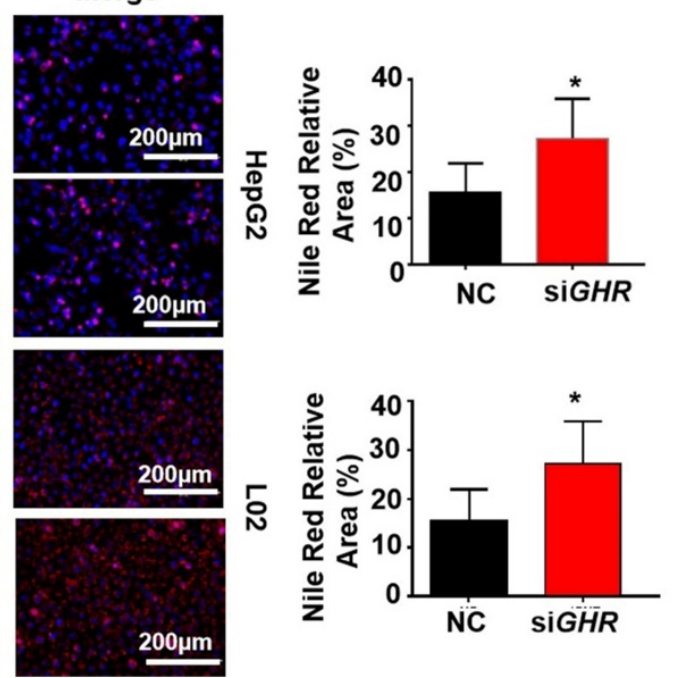

Figure 4. GHR depletion caused intracellular lipid accumulation in cultured human hepatocytes. (A-E) Hepatocytes were treated with NC or GHR siRNA (siGHR). (A-B) The GHR protein expression levels in human hepatocytes, and quantification of the content by Imagej. (C) TG levels in human hepatocytes. (D) Nile red staining of human hepatocytes with NC or siGHR. (E) The neutral lipid content was quantified with Imagej and normalized to the number of nuclei. Scale bar: $100 \mu \mathrm{m}$. $\mathrm{n}=3$ per group. The data are presented as the mean \pm SD values. $*, P<0.05 ; * *, P<0.01 ; * * *, P<0.001$

\section{Knockdown of GHR causes intracellular lipid accumulation in cultured human hepatocytes}

To explore whether hepatic steatosis caused by GHR KO in pigs also occurs in humans, siGHR was transfected into two human hepatocyte lines, HepG2 and L02, to silence GHR. Western blot analysis showed that GHR expression was markedly decreased in siGHR-treated human hepatocytes (Figure 4A). The mRNA levels of genes responsible for fatty acid oxidation were significantly downregulated in siGHR-treated human hepatocytes (Supplementary Figure 3B). Consistent with the results in GHR KO pigs, no significant difference was detected in the mRNA levels of genes related to fatty acid transport and synthesis in siGHR-treated human hepatocytes (Supplementary Figure 3B). The level of intracellular TGs was increased in siGHR human hepatocytes (Figure 4C), and Nile red staining indicated that lipid deposition was increased in siGHR human hepatocytes (Figure 4D-F). As a comparison, we also conducted similar experiments on siGhr mouse hepatocytes. Quantitative real-timePCR (Q-PCR) showed that Ghr expression was markedly decreased in siGhr Hepa1-6 compared with control cells (Supplementary Figure 5A). Unlike the results in humans and pigs, there were no significant changes in the mRNA levels of genes related to fatty acid oxidation in siGhr Hepa1-6 cells (Supplementary Figure 3C). Moreover, the levels of intracellular TGs (Supplementary Figure 5B) and Nile red staining (Supplementary Figure 5C-D) were not significantly different in siGhr mouse hepatocytes. These results indicate that knockdown of GHR leads to intracellular lipid accumulation in human hepatocytes but not in mice. Taken together, we conclude that GHR KO pigs more accurately mimic abnormal lipid metabolism in LS patients.

\section{Transcriptional profile of GHR KO pigs during hepatic steatosis}

To explore the causes of hepatic steatosis resulting from GHR KO, we performed RNAseq analysis on liver tissue from GHR KO pigs. With the cut-off criteria of a false discovery rate $<0.05$ and Fold Change $\geq 2,897$ (306 upregulated, 591 downregulated) differentially expressed genes (DEGs) were identified in GHR KO pigs (Supplementary Figure 6A-B). Gene 
ontology (GO) term and Kyoto Encyclopedia of Genes and Genomes (KEGG) pathway analyses were performed with the lipid metabolism-related genes. GO terms (Figure 5A) related to lipid oxidation, sphingolipid biosynthetic process, and lipid metabolic process and KEGG pathways (Figure 5B) of fatty acid metabolism and fatty acid degradation were enriched. The transcriptome data also identified some differentially expressed transcription factors (TFs), among which AHR, an important transcription factor in the regulation of lipid metabolism, was significantly downregulated in GHR KO pigs (Supplementary Figure 6C). The mRNA and protein levels of AHR were significantly reduced in GHR KO pigs and siGHR human hepatocytes (Figure 5C-E, I-M), whereas the expression of Ahr was not changed in siGhr mouse hepatocytes (Figure 5F-H). These results suggest that AHR misregulation may be responsible for lipid accumulation in humans and pigs.
A

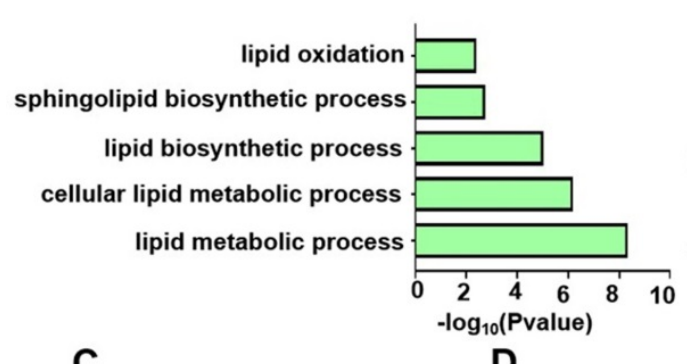

C
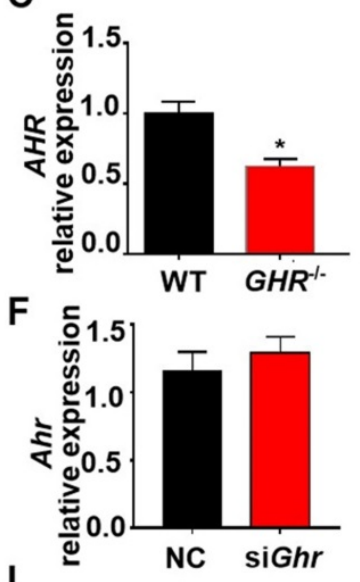

|

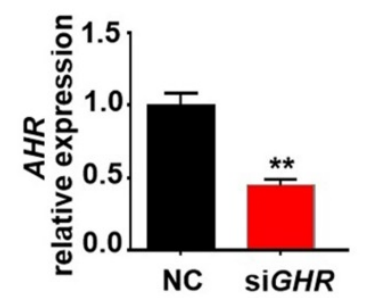

$\mathbf{L}$
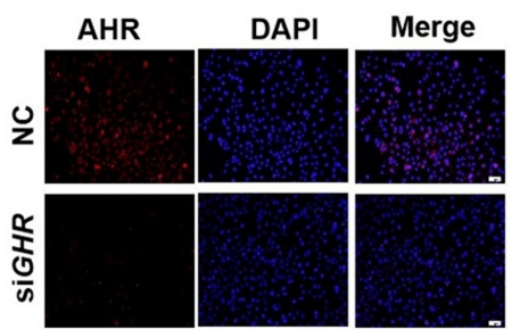

D

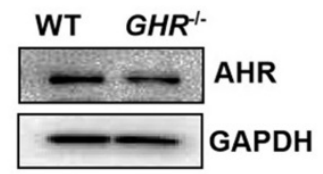

G

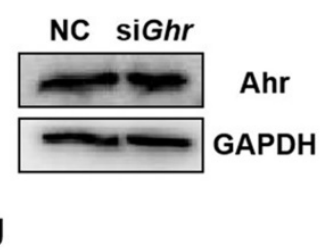

B

\section{KEGG Enrichment}

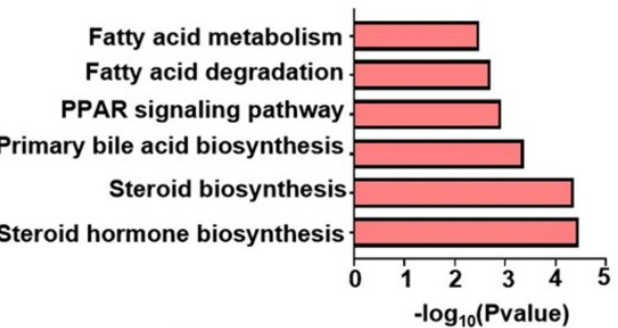

E

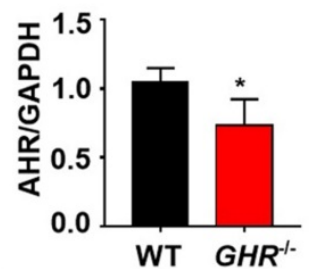

H
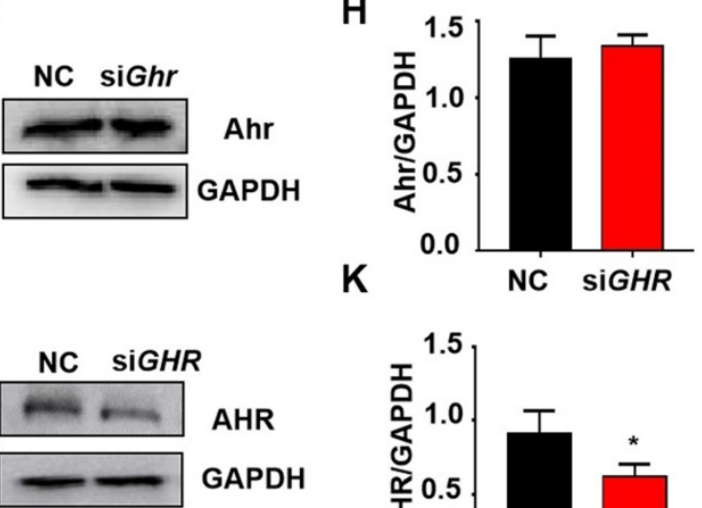

AHR

GAPDH
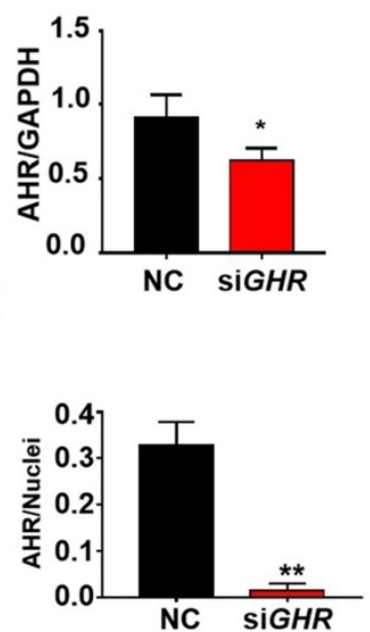

Figure 5. Transcriptome analysis of hepatic gene expression profiles in GHR KO pigs. (A) The five enriched biological processes contributing to GHR function were determined by GO analysis based on the DEGs. (B) KEGG pathway enrichment analysis of the six identified lipid metabolism-related processes. (C-E) mRNA and protein levels of AHR in the livers of WT and GHR KO pigs. (F-H) mRNA and protein levels of AHR in NC and siGhr mouse hepatocytes. (I-M) mRNA and protein levels of AHR in NC and siGHR human hepatocytes. (I) mRNA levels of AHR in NC and siGHR human hepatocytes. (J-K) Protein levels of AHR in NC and siGHR human hepatocytes by Western blotting and quantification of the content by Imagej. (L-M) Protein levels of AHR in NC and siGHR human hepatocytes by immunofluorescence and quantification of the content by Imagej. Scale bar: $100 \mu \mathrm{m} . \mathrm{n}=3$ per group. The data are presented as the mean $\pm S D$ values. $*, P<0.05 ; * *, P<0.01$; ***, $P<0.001$. 
A
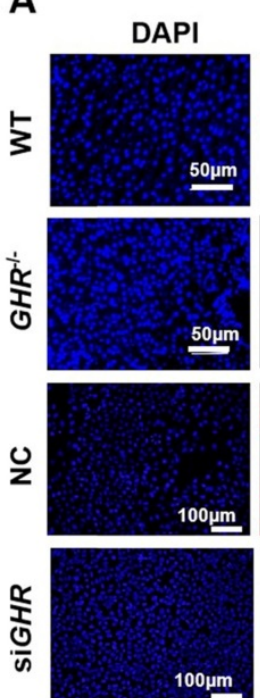

C

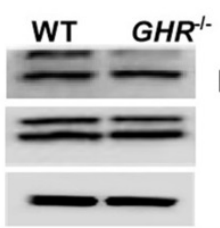

$100 \mu \mathrm{m}$

soum
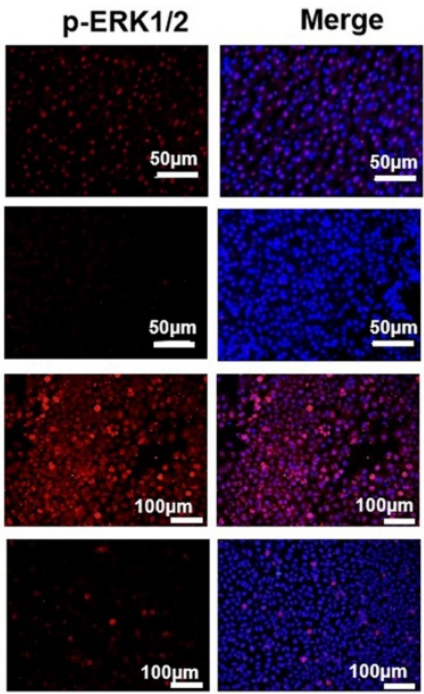

B
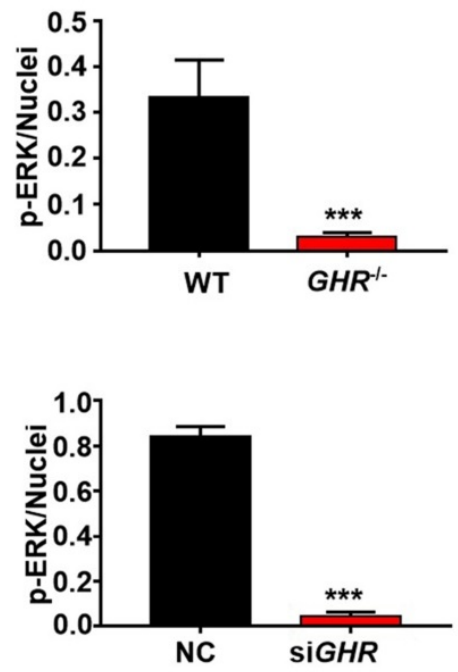

D

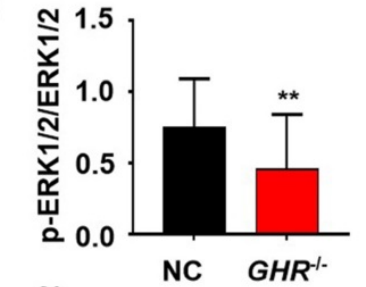

NC SIGHR

p-ERK1/2

ERK1/2

GAPDH

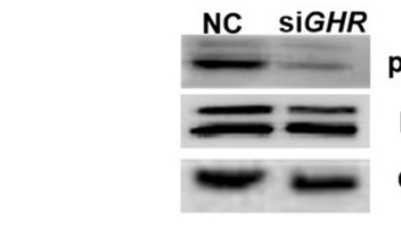

p-ERK1/2

ERK1/2

GAPDH

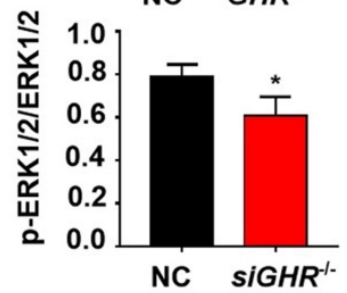

E

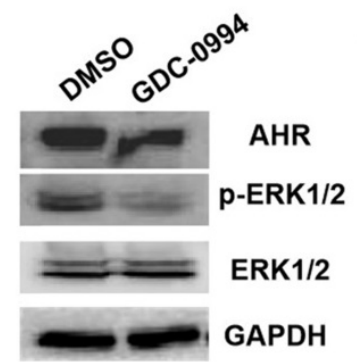

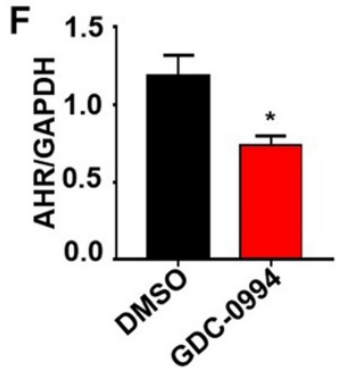

G

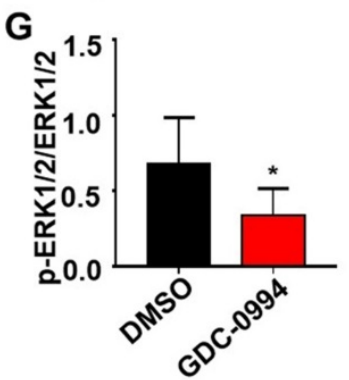

Figure 6. GHR regulates AHR expression through the MAPK signaling pathway. (A-B) IF staining of p-ERK1/2 in pigs and hepatocytes, and quantification of the content by ImageJ. (C-D) Protein levels of P-ERK1/2 and ERK1/2 in pigs and hepatocytes by Western blotting and quantification of the content by Imagej. (E-G) Protein levels of AHR, p-ERK 1/2 and ERK1/2 in L02 cells treated with GDC-0994 (an ERK inhibitor), and quantification of the content by Image. Scale bar $=50 \mu \mathrm{m}$. $\mathrm{n}=3 \mathrm{per}$ group. The data are presented as the mean \pm SD values. *, $\mathrm{P}<0.05$; **, $\mathrm{P}<0.01$; ***, $\mathrm{P}<0.001$.

\section{GHR regulates AHR expression through the MAPK/ERK signaling pathway}

The above results show that by interfering with the expression of GHR, AHR is significantly downregulated. To explore the mechanism of AHR involvement in hepatic steatosis caused by GHR deletion, we used Co-IP experiments to verify whether there is a protein interaction between AHR and GHR. The results showed that AHR did not directly interact with GHR (Supplementary Figure
7A-B). Given that MAPK is the classical signaling pathway downstream of GHR and the confirmed crosstalk between the MAPK and AHR pathways, we speculated that GHR may influence the expression of AHR through MAPK. First, immunofluorescence results showed that the phosphorylation levels of ERK1/2 were significantly decreased in both GHR KO pigs and siGHR human hepatocytes (Figure 7A-B). Western blot findings confirmed this conclusion (Figure 6C-D). Furthermore, Western blot analysis confirmed that blocking the function of ERK1/2 with 
GDC-0994 resulted in the downregulation of AHR expression (Figure 6E-G). These results suggest that GHR regulates the expression of AHR through the MAPK/ERK signaling pathway.

\section{AHR directly activates fatty acid oxidation gene expression}

To investigate the relationship between the expression of AHR and fatty acid oxidation in hepatic steatosis induced by GHR deletion, we examined the expression of genes related to fatty acid oxidation. The protein levels of ACOX1 and CPT1A in human hepatocytes were increased after the addition of Tapinarof (an AHR ligand), while, AHR knockdown significantly decreased Tapinarof-induced ACOX1 and CPT1A expression at protein level (Figure 7A-B).
To assess whether ACOX1 and CPT1A are directly regulated by $A H R$, we checked their activity in hepatocytes following Tapinarof treatment. The luciferase reporter assay manifested that stimulating the AHR with Tapinarof promoted the activity of ACOX1 and CPT1A, whereas AHR knockdown by siAHR treatment had the opposite effect (Figure 7C-D). Next, chromatin immunoprecipitation (ChIP) assays and real-time PCR analysis of the immunoprecipitated DNA demonstrated that AHR directly bound to the promoter regions of CPT1A and ACOX1 (Figure 7E). The above results indicate that AHR directly activates the expression of fatty acid oxidation genes at the transcriptional level. To explore whether the protective effects of GHR are dependent on AHR in hepatic steatosis, we carried out the rescue

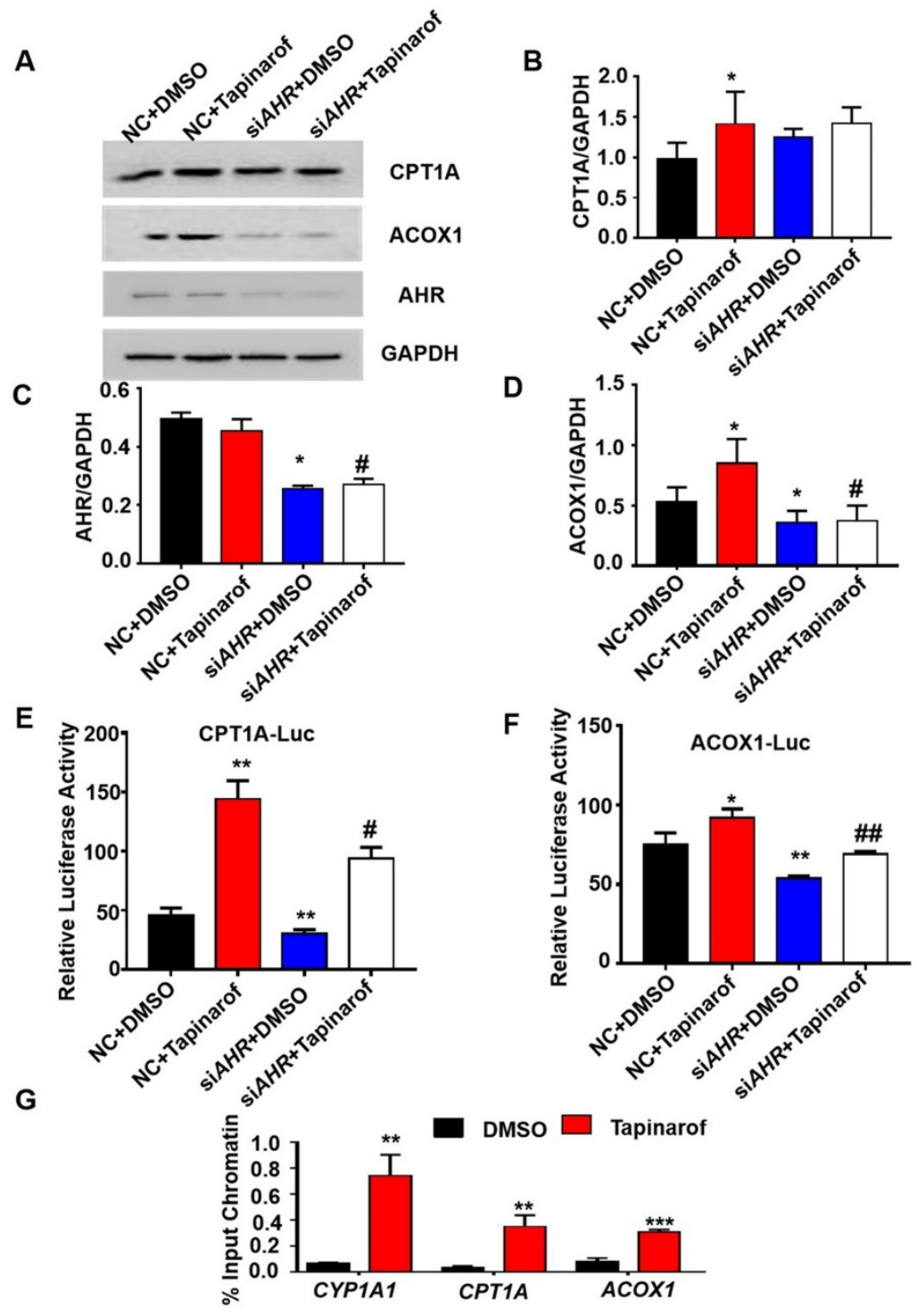

Figure 7. AHR directly activated fatty acid oxidation gene expression. (A-D) Protein levels of CPTIA, ACOX1 and AHR in cells treated with Tapinarof (an AHR ligand) and/or $A H R$ siRNA (siAHR), and quantification of the content by Imagel. (E) Effect of Tapinarof and/or siAHR treatment on ACOXI luciferase reporter activity. (F) Effect of Tapinarof and/or siAHR treatment on CPTIA luciferase reporter activity. (G) ChIP assays were performed with the CYPIAI, CPTIA and ACOXI promoters in L02 cells with or without Tapinarof $(10 \mu \mathrm{M})$ treatment for 12 h. $\mathrm{n}=3$ per group. The data are presented as the mean \pm SD values. $*, P<0.05 ; * *, P<0.01 ; * * *, P<0.001$ compared with the first group, \#, $\mathrm{P}<0.05 ; \#, \mathrm{P}<0.01 ; \ldots+\cdots, \mathrm{P}<0.001$ compared with the second group. 
experiments on siGHR human hepatocytes. The results of TG measurement and Nile red staining showed that overexpression of AHR could alleviate lipid deposition induced by GHR deletion (Figure 8A-B).

\section{Discussion}

Here we report a large animal model of LS caused by GHR deficiency. Most previous studies have used the Ghr KO mouse model to explore the function of GH and have provided new insights into the consequences of $\mathrm{GH}$ insensitivity for metabolic functions [27]. Ghr KO mice showed increased serum levels of GH and decreased serum levels of IGF-I, obesity and hypoglycaemia $[13,27,28]$. However, the influence of GHR mutation on lipid metabolism differs between mice and humans. The serum levels of FFAs in Ghr KO mice are reduced, while individuals with LS usually show increased FFA levels [27]. Compared with mice, minipigs are similar to humans in terms of lipid metabolism [18,19]. Therefore, we used gene editing technology to successfully knock out the GHR gene in pigs and establish the GHR KO model of LS; these animals showed typical dwarfism symptoms and obesity [22]. Interestingly, unlike the reduced level of FFAs in Ghr KO mice and GHR KO pigs $[27,29]$, the serum level of FFAs in our GHR KO pigs was significantly increased, which was consistent with the higher serum level of FFAs in LS patients [30]. Therefore, our GHR KO pigs could more accurately mimic lipid metabolism in human LS.

As the central organ in metabolism, the liver participates in a variety of lipid metabolic pathways. Fatty liver begins with the deposition of TGs in hepatocytes and the formation of lipid droplets of different sizes. The increases in fat decomposition, the serum level of FFAs and fat synthesis and the decrease in fatty acid oxidation result in hepatic lipid accumulation [31-34]. Patients with LS often tend to develop fatty livers [9]. Interestingly, previous studies have found no hepatic steatosis in global Ghr KO mice [11], except liver-specific Ghr KO mice [35]. However, our new observations revealed an important role of GHR deficiency in promoting hepatic steatosis. In our study, both GHR KO pigs and siGHR human hepatocytes manifested lipid accumulation and reduced fatty acid oxidation, which was not observed in siGhr mouse hepatocytes. Therefore, reduced fatty acid oxidation may be an important cause of hepatic steatosis due to GHR deficiency. The above results further indicate that pigs can better simulate the symptoms of abnormal lipid metabolism in humans.

Inflammation is an important indicator of the development of liver steatosis to liver fibrosis [36]. Unfortunately, due to the limitation of experimental conditions, we did not obtain experimental pigs of greater monthly age. Therefore, the current inflammation-related indicators of GHRKO pigs have not changed significantly, follow-up experiments are needed to explore in future.
A
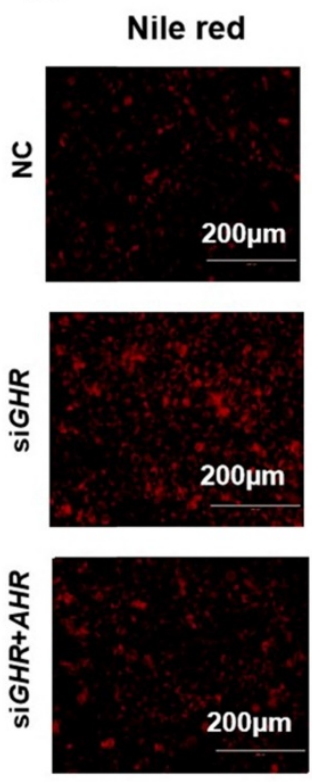

DAPI
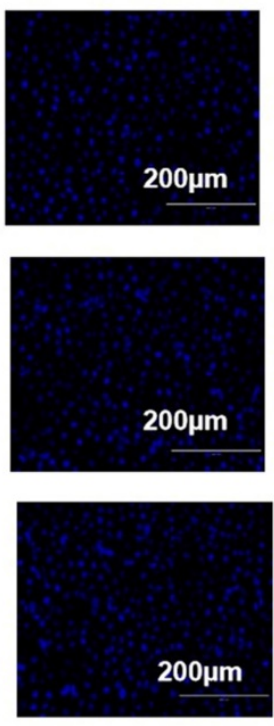

Merge
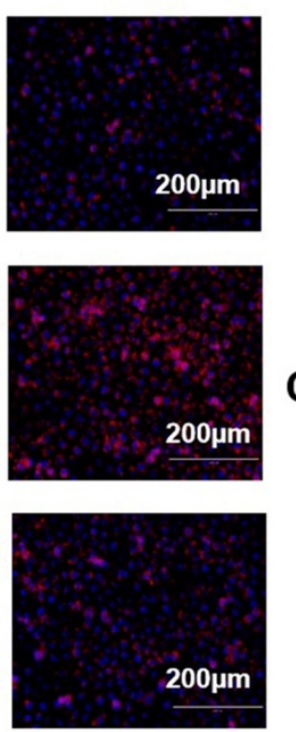

B

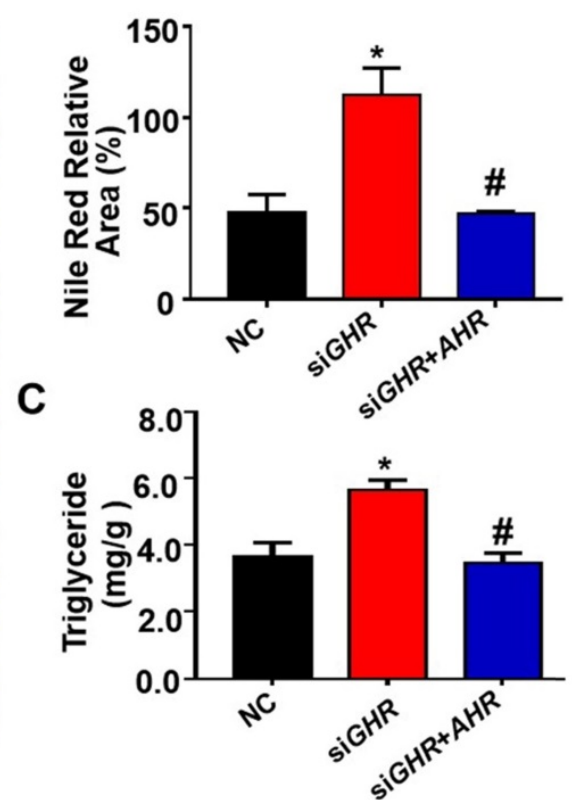

Figure 8. Overexpression of AHR alleviated lipid deposition induced by GHR deletion. (A) Nile red staining and (C) TG content in NC and siGHR cells with or without PCMV-Myc-AHR transfection. (B) The neutral lipid content was quantified with Imagej and normalized to the number of nuclei. The data are presented as the mean \pm SD values. Scale bar: $100 \mu \mathrm{m} . \mathrm{n}=3$ per group. *, $\mathrm{P}<0.05$; **, $\mathrm{P}<0.01$; ***, $\mathrm{P}<0.001$ compared with the first group; $\#$, $\mathrm{P}<0.05$; \#, $\mathrm{P}<0.01$; \#\#, $\mathrm{P}<0.001$ compared with the second group. 

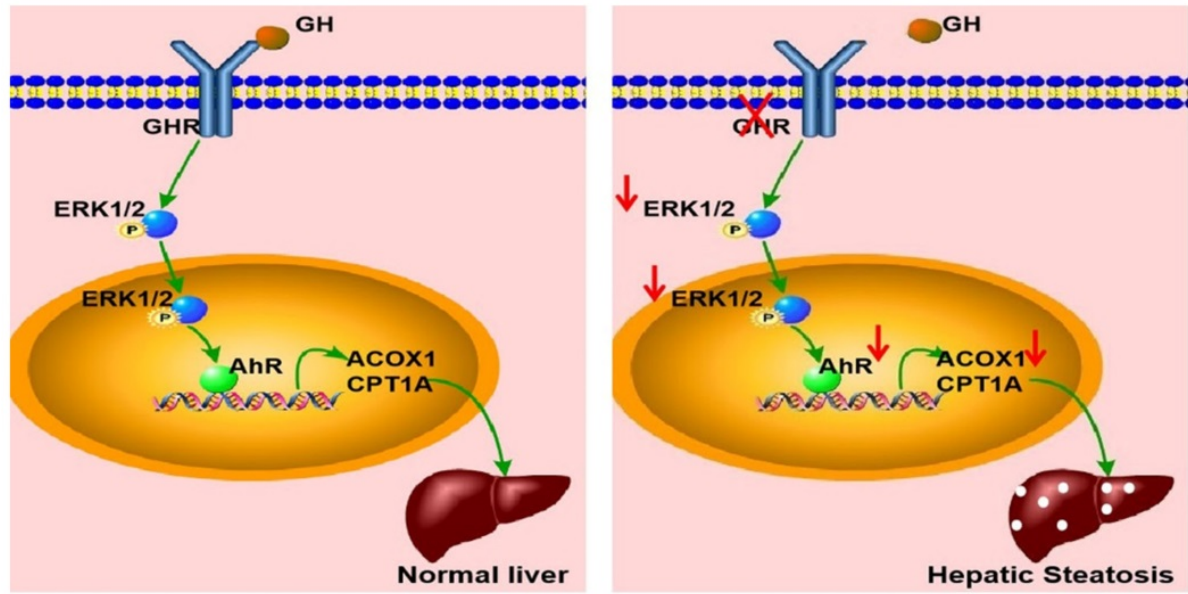

Figure 9. Schematic illustration of the proposed role of GHR in hepatic steatosis. Normal liver (left): GH binds to GHR and promotes the expression of AHR by activating ERK $1 / 2$ via phosphorylation. Activated AHR directly binds to the promoter regions of ACOXI and CPTIA to transcriptionally activate their expression and allow normal fatty acid oxidation function. Hepatic steatosis (right): Loss of GHR reduces the expression of AHR by reducing the phosphorylation level of ERK1/2. AHR downregulation directly reduces fatty acid oxidation, leading to hepatic steatosis.

Based on the analysis of our transcriptome data, 23 transcription factors were identified, and the transcription factor AHR was identified as one of the top hits, which has been linked to hepatic steatosis in previous reports. AHR can affect hepatic glucose and lipid metabolism in either a detrimental or beneficial manner $[37,38]$. Some studies have shown that CD36, a key gene in fatty acid transport [39], is a new transcription target for AHR. Activation of AHR can induce CD36 expression, enhance fatty acid uptake, and lead to hepatic steatosis [40]. However, others have found that treatment with AHR agonists can reduce the synthesis of fatty acids [41]. While the mRNA and protein levels of Ahr in siGHR mouse hepatocytes were normal, our results showed that the mRNA and protein levels of AHR in GHR KO pigs were reduced, and knockdown of GHR in human hepatocytes led to a significant downregulation of AHR, resulting in lipid accumulation, and that overexpression of $A H R$ ameliorated lipid accumulation. Furthermore, we found that AHR could bind directly to the promoter regions of $A C O X 1$ and $C P T 1 A$ (the key genes in fatty acid oxidation) and transcriptionally activate their expression.

In summary, GHR KO pigs showed abnormal lipid metabolism, especially high serum levels of FFAs and hepatic steatosis, consistent with the results observed in LS patients. A key mediator of this effect appears to be that GHR deficiency in hepatocytes inhibited the expression of AHR by decreasing the phosphorylation level of ERK1/2. Moreover, we confirmed that $A C O X 1$ and $C P T 1 A$ were direct target genes of $A H R$. The reduction in AHR expression directlyled to a reduction in fatty acid oxidation, an important cause of hepatic steatosis in the context of GHR deficiency (Figure 9). Thus, AHR in hepatocytes is a critical factor in the initiation of hepatic steatosis and a potential molecular target for intervention.

\section{Abbreviations}

LS: Laron syndrome; GHR: growth hormone receptor; FFAs: free fatty acids; ZFN: zinc finger nuclease; CDS: coding sequence; NC: negative control; HOMA-IR: homeostatic model assessment of insulin resistance; HOMA-IS: homeostatic model assessment of insulin sensitivity; TGs: triglycerides; TC: total cholesterol; HDL: high-density lipoprotein; LDL: low-density lipoprotein; ALT: alanine aminotransferase; AST: aspartate aminotransferase; DEGs: differentially expressed genes; GO: Gene Ontology; KEGG: Kyoto Encyclopedia of Genes and Genomes; TFs: transcription factors; ChIP: chromatin immunoprecipitation; Co-IP: Co-Immunoprecipitation.

\section{Supplementary Material}

Supplementary figures and table.

http://www.ijbs.com/v17p4108s1.pdf

\section{Acknowledgements}

This study was supported by grants 2016ZX08006002-003, 2014ZX08006-004, 2013ZX08006-002 and 2009ZX08006-008B from the National Transgenic Major Program. The authors would like to thank Ronghui Li at the Salk Institute for Biological Studies for his help in revising the manuscript.

\section{Ethics approval}

All procedures involving animals were approved by the Laboratory Animal Welfare and Animal Experiment Ethics Review Committee of China Agricultural University (No. Aw42601202-3-1) 
and in accordance with the NIH Guide for the Care and Use of Laboratory Animals.

\section{Data availability}

All data are available within the article or Supplementary Information. The RNAseq data reported in this paper are available in NCBI BioProject ID: PRJNA739087. Source data are provided with this paper.

\section{Author Contributions}

Author contributions are as follows. Q.H., H.L.C., L.K.W. and Y.A. contributed to the performance of experiments and data analysis; Q.H. wrote the original manuscript; R.Z. provided conceptualization, wrote the original manuscript and reviewed the manuscript; X.X.H., Y.F.Z. and H.Z. reviewed the manuscript.

\section{Competing Interests}

The authors have declared that no competing interest exists.

\section{References}

1. Lin S, Li C, Li C, Zhang X. Growth Hormone Receptor Mutations Related to Individual Dwarfism. Int J Mol Sci. 2018; 19:1433.

2. Li R, Gong F, Pan H, Liang H, Miao H, Zhao Y, et al. Identification and In vitro Functional Verification of Two Novel Mutations of GHR Gene in the Chinese Children with Laron Syndrome. Front Endocrinol (Lausanne). 2021; 12:605736.

3. Alessia D, Vivian H, Metherell L A, Irène N, Cecilia C H, Clark A, et al Evidence for a continuum of genetic, phenotypic, and biochemical abnormalities in children with growth hormone insensitivity. Endocr. Rev. 2011; 32:472-494.

4. Brooks A J, Wooh J W, Tunny K A, Waters M J. Growth hormone receptor; mechanism of action. Int J Biochem Cell B. 2008; 40:1984-1989.

5. Vanderkuur J A, Butch E R, Waters S B, Pessin J E, Kun-Liang G, Christin C S. Signaling molecules involved in coupling growth hormone receptor to mitogen-activated protein kinase activation. Endocrinology. 1997; 138:4301-4307.

6. Gao S, Ni O, Wu X, Cao T. GHR knockdown enhances the sensitivity of HCC cells to sorafenib. Aging. 2020; 12:18127-18136.

7. Eidne K A, Parker M W, Brown R J, Adams J J, Pelekanos R A, Wan Y, et al. Model for growth hormone receptor activation based on subunit rotation within a receptor dimer. Nat Struct Mol Biol. 2005; 12:814-821.

8. Tao Z, Goh E, Graichen R, Ling L, Lobie P E. Signal transduction via the growth hormone receptor. Cell Signal. 2001; 13:599-616.

9. Laron Z, Ginsberg S, Webb M. Nonalcoholic fatty liver in patients with Laron syndrome and GH gene deletion - preliminary report. Growth Horm IGF Res. 2008; 18:434-438.

10. List E O, Berryman D E, Buchman M, Parker C, Funk K, Bell S, et al. Adipocyte-specific $\mathrm{GH}$ receptor null (AdGHRKO) mice have enhanced insulin sensitivity with reduced liver triglycerides. Endocrinology. 2018; 160:68-80.

11. Sun L Y, Bartke A. Tissue-Specific GHR Knockout Mice: Metabolic Phenotypes. Front Endocrinol (Lausanne). 2014; 5:243.

12. List E O, Berryman D E, Funk K, Jara A, Kelder B, Wang F, et al. Liver-Specific GH Receptor Gene-Disrupted (LiGHRKO) Mice Have Decreased Endocrine IGF-I, Increased Local IGF-I, and Altered Body Size, Body Composition, and Adipokine Profiles. Endocrinology. 2014; 155:1793-1805.

13. Berryman D E, List E O, Palmer A J, Chung M Y, Wright-Piekarski J, Lubbers E, et al. Two-year body composition analyses of long-lived GHR null mice. J Gerontol A Biol Sci Med Sci. 2010; 65:31-40.

14. Guo Y, Lu Y, Houle D, Robertson K, Tang Z, Kopchick J J, et al. Pancreatic islet-specific expression of an insulin-like growth factor-I transgene compensates islet cell growth in growth hormone receptor gene-deficient mice. Endocrinology. 2005; 146:2602-2609.
15. Duran-Ortiz S, Noboa V, Kopchick J J. Tissue-specific disruption of the growth hormone receptor (GHR) in mice: An update. Growth Horm IGF Res. 2019; 51:1-5.

16. Hinrichs A, Kessler B, Kurome M, Blutke A, Kemter E, Bernau M, et al. Growth hormone receptor-deficient pigs resemble the pathophysiology of human Laron syndrome and reveal altered activation of signaling cascades in the liver. Mol Metab. 2018; 11:113-128.

17. Casellas J, Vidal O, Pena R N, Gallardo D, Manunza A, Quintanilla R, et al. Genetics of serum and muscle lipids in pigs. Anim Genet. 2013; 44:609-619.

18. Zhang W, Su W, Li P, Rao H, Lin Q, Zeng X, et al. Naoxintong Capsule Inhibits the Development of Cardiovascular Pathological Changes in Bama Minipig Through Improving Gut Microbiota. Front Pharmacol. 2019; $10: 1128$

19. Johansen T, Hansen H S, Richelsen B, Malmlöf R. The obese Göttingen minipig as a model of the metabolic syndrome: dietary effects on obesity, insulin sensitivity, and growth hormone profile. Comparative Med. 2001; 51:150-155.

20. Muir W M. Relative Efficiency of Selection for Performance of Birds Housed in Colony Cages Based on Production in Single Bird Cages. J Transl Med. 1985; 13:1-12.

21. Schumacher-Petersen C, Christoffersen B Ø, Kirk R K, Ludvigsen T P, Zois N E, Pedersen H D, et al. Experimental non-alcoholic steatohepatitis in Göttingen Minipigs: consequences of high fat-fructose-cholesterol diet and diabetes. J Transl Med. 2019; 17:110.

22. Cui D, Li F, Li Q, Li J, Zhao Y, Hu X, et al. Generation of a miniature pig disease model for human Laron syndrome. Sci Rep. 2015; 5:15603.

23. Bartke A, Sun L Y, Longo V. Somatotropic Signaling: Trade-Offs Between Growth, Reproductive Development, and Longevity. Physiol Rev. 2013; 93:571-598.

24. Bartke A. Growth hormone, insulin and aging: The benefits of endocrine defects. Exp Gerontol. 2011; 46:108-111.

25. Schultze S M, Hemmings B A, Niessen M, Tschopp O. PI3K/AKT, MAPK and AMPK signalling: protein kinases in glucose homeostasis. Expert Rev. Mol. Med. 2012; 14:1.

26. Cheng C, Zhuo S, Zhang B, Zhao X, Liu Y, Liao C, et al. Treatment implications of natural compounds targeting lipid metabolism in nonalcoholic fatty liver disease, obesity and cancer. Int J Biol Sci. 2019; 15:1654-1663.

27. List E O, Sackmann-Sala L, Berryman D E, Funk K, Kelder B, Gosney E S, et al. Endocrine parameters and phenotypes of the growth hormone receptor gene disrupted (GHR-/-) mouse. Endocr Rev. 2011; 32:356-386.

28. Zhou Y, Xu B C, Maheshwari H G, He L, Reed M, Lozykowski M, et al. A mammalian model for Laron syndrome produced by targeted disruption of the mouse growth hormone receptor/binding protein gene (the Laron mouse). P Natl Acad Sci Usa. 1997; 94:13215-13220.

29. Riedel E O, Hinrichs A, Kemter E, Dahlhoff M, Backman M, Rathkolb B, et al. Functional changes of the liver in the absence of growth hormone (GH) action-Proteomic and metabolomic insights from a GH receptor deficient pig model. Mol Metab. 2020; 36:100978.

30. Laron Z. Laron syndrome (primary growth hormone resistance or insensitivity): the personal experience 1958-2003. J Clin Endocrinol Metab. 2004; 89:1031-1044.

31. Byrne C D, Targher G. NAFLD: a multisystem disease. J Hepatol. 2015; 62: S47-S64

32. Alves-Bezerra M, Cohen D E. Triglyceride Metabolism in the Liver. Compr Physiol. 2017; 8:1-8.

33. Brunt E M, Wong W S, Nobili V, Day C P, Rinella M E. Nonalcoholic fatty liver disease. Nat Rev Dis Primers. 2015; 1:15080.

34. Xu M, Wu H, Li M, Wen Y, Yu C, Xia L, et al. DJ-1 Deficiency Protects Hepatic Steatosis by Enhancing Fatty Acid Oxidation in Mice. Int J Biol Sci. 2018; 14:1892-1900.

35. Fan Y, Menon R K, Cohen P, Hwang D, Clemens T, DiGirolamo D J, et al. Liver-specific deletion of the growth hormone receptor reveals essential role of growth hormone signaling in hepatic lipid metabolism. J Biol Chem. 2009; 284:19937-19944.

36. Nobili V, Alisi A, Valenti L, Miele L, Feldstein A E, Alkhouri N. NAFLD in children: new genes, new diagnostic modalities and new drugs. Nat Rev Gastroenterol Hepatol. 2019; 16:517-530.

37. Xyzab C, Hgxab C, Zhwab C, Blab C, Hyjab C, Dllab C, et al. In vitro and in vivo approaches for identifying the role of aryl hydrocarbon receptor in the development of nonalcoholic fatty liver disease. Toxicol Lett. 2020; 319:85-94.

38. Biljes D, Hammerschmidt-Kamper C, Kadow S, Diel P, Esser C. Impaired glucose and lipid metabolism in ageing aryl hydrocarbon receptor deficient mice. Excli J. 2015; 14:1153-1163.

39. Zhao L, Zhang C, Luo X, Wang P, Zhou W, Zhong S, et al. CD36 palmitoylation disrupts free fatty acid metabolism and promotes tissue 
inflammation in non-alcoholic steatohepatitis. J Hepatol. 2018; 68:705-717.

40. Lee J H, Wada T, Febbraio M, He J, Matsubara T, Lee M J, et al. A Novel Role for the Dioxin Receptor in Fatty Acid Metabolism and Hepatic Steatosis. Gastroenterology. 2010; 139:653-663.

41. Rachel T, Murray I A, Smith P B, Andrew P, Perdew G H. Role of the Ah Receptor in Homeostatic Control of Fatty Acid Synthesis in the Liver. Toxicol Sci. 2012; 129:372-379. 